Article

\title{
Watermelon Rind and Flesh Volatile Profiles and Cultivar Difference
}

\author{
Xiaofen Du *D and Jessica Ramirez
}

Citation: Du, X.; Ramirez, J.

Watermelon Rind and Flesh Volatile

Profiles and Cultivar Difference.

Horticulturae 2022, 8, 99. https://

doi.org/10.3390/horticulturae8020099

Academic Editors: Christina Dorado, Wei Zhao and Xiuxiu Sun

Received: 23 December 2021

Accepted: 20 January 2022

Published: 23 January 2022

Publisher's Note: MDPI stays neutral with regard to jurisdictional claims in published maps and institutional affiliations.

Copyright: (C) 2022 by the authors. Licensee MDPI, Basel, Switzerland. This article is an open access article distributed under the terms and conditions of the Creative Commons Attribution (CC BY) license (https:// creativecommons.org/licenses/by/ $4.0 /)$.
Department of Nutrition and Food Sciences, Texas Woman's University, Denton, TX 76204, USA; jramirez37@twu.edu

* Correspondence: xdu@twu.edu; Tel.: +1-940-898-2667

\begin{abstract}
Watermelon rind is treated as agricultural waste and commonly discarded, causing environmental issues and biomass loss. This study aimed to identify volatile profiles of watermelon rind and flesh and their cultivar difference. Volatiles were analyzed using solid-phase microextraction-gas chromatography-mass spectrometry (SPME-GC-MS). A total of 132 volatiles were identified, including aldehydes, alcohols, ketones, terpenes/terpenoids, esters, lactones, acids, and sulfides. In both rind and flesh, the most dominant compounds in numbers and abundance (peak area) were aldehydes and alcohols, which accounted $94-96 \%$ of the total volatile abundance in the rind and $85-87 \%$ in the flesh. Total volatile in watermelon rind was only $28-58 \%$ of the corresponding flesh samples. Both rind and flesh shared nine-carbon aldehydes and alcohols, though the rind lacked additional diversity. Volatile difference between rind and flesh was greater than the difference among cultivars, although volatiles in the rind could be two times difference between Fascination and other three watermelons (Captivation, Exclamation, and Excursion). This study provides the first-hand knowledge regarding watermelon rind volatile profiles and cultivar difference and shows the potential use of rind in food or beverages due to its naturally contained nine-carbon compounds.
\end{abstract}

Keywords: Citrullus lanatus; watermelon rind; rind volatile; watermelon flesh; watermelon flavor

\section{Introduction}

Watermelon (Citrullus lanatus) is globally consumed, possessing unique organoleptic properties, hydration function, and well-studied health benefits owed to its red flesh, though verities with orange and yellow flesh also produced [1]. Roughly, watermelon consists of skin, rind, flesh, and seeds. Watermelon flesh accounts for approximately $60 \%$ of the total watermelon mass [2]. Watermelon flesh contains a large amount of water, which is approximately $93 \%$ of the total weight of the flesh [3]; it also contains micronutrients such as vitamins ( $\mathrm{A}, \mathrm{B}, \mathrm{C}, \mathrm{E})$, mineral salts $(\mathrm{K}, \mathrm{Mg}, \mathrm{Ca}, \mathrm{Fe})$, amino acids (citrulline and arginine), lycopene, and phenolic compounds [2].

The edible rind makes up approximately $40 \%$ of the total watermelon mass yet is often discarded as waste [4]. Direct disposal of the rind waste is causing environmental issues, though several approaches of reusing watermelon rind have been investigated in a laboratory scale. The specialized function of the rind's polysaccharide composition (pectin and fiber) has been considered a potential reason for its reuse [2,5]. It would be favorable to take advantage of the nutritional potential of rind and create commercial value, rather than limiting it to agricultural waste. Approaches have been introduced to reduce the accumulation of solid watermelon waste by converting the rind's polysaccharides into other products such as biosorbent [6,7], bioremediation [8], biochar [9], and bioethanol [4] Additionally, watermelon rind has been studied as a source of nutritional food ingredients such as antioxidants [10], amino acids [11-14], and pectin [15,16], especially citrulline. In processed foods, rind has been tested in pickled form and in jam $[17,18]$. Watermelon rind in powder form has been examined to apply in carbohydrate-based goods including 
cakes [12,19], cookies [20,21], noodles [22,23], beef patties [24], and pork patties [25]. Furthermore, a few studies have investigated watermelon rind as a possible growth medium for microbials [26,27].

Though the chemical composition of watermelon rind has been studied, its volatile profile has not yet been reported. Watermelon aroma is derived from volatile composition, playing a key role in flavor quality. The most abundant volatiles in watermelon flesh are sixcarbon and nine-carbon volatiles. Nine-carbon saturated or unsaturated linear aldehydes and alcohols are the best representation of watermelon aroma, which include (Z)-2-nonenal, (E)-2-nonenal, (E,Z)-2,6-nonadienal, (Z)-3-nonenol, (E)-6-nonenol, (E,Z)-3,6-nonadienol, (E,E)-3,6-nonadienol, and (Z,Z)-3,6-nonadienol [28]. These nine-carbon compounds are derived from fatty acids via the lipoxygenase metabolic pathway [29]. The volatile profiles indicated variety difference [29], though limited studies have focused on watermelon volatile variety difference.

Our previous study shows watermelon variety difference based on their sensory qualities [30]; however, volatiles in these varieties have not been investigated yet. It is unknown if volatile difference between flesh and rind exists and if volatiles in watermelon rind show a variety difference. Understanding volatile composition in watermelon rind will provide evidence for potential uses of watermelon rind in food and beverages. Therefore, this study aimed to (1) identify volatile compounds in watermelon rinds and the corresponding flesh, and (2) investigate part difference (rind vs. flesh) and volatile variety difference among four watermelon varieties.

\section{Materials and Methods}

\subsection{Watermelon Samples}

The watermelon cultivars (Captivation, Exclamation, Excursion, and Fascination) used previously in descriptive sensory analysis [30], were subjected to volatile analysis in this study. The four watermelon cultivars were picked from the Texas A\&M Agrilife Research and Extension Center at Lubbock, Texas (33.6935 ${ }^{\circ}$ N, $101.8249^{\circ}$ W) in September 2019, where at least six watermelon fruits per cultivar were randomly harvested. One melon $(\sim 6.0 \mathrm{~kg})$ of each of the four cultivars was prepared by washing with tap water, drying with paper towels, and peeling with a knife. The entire flesh (red colored portion of the fruit, $\sim 3.5 \mathrm{~kg} /$ melon) of each melon was diced into $1 \mathrm{~cm}^{3}$ pieces using a knife and mixed thoroughly using a spatula to ensure homogeneity. Separately, watermelon rinds $(\sim 1 \mathrm{~cm}$ thick green/white colored outer edge portion of the fruit, $\sim 0.8 \mathrm{~kg} / \mathrm{melon}$, with the steaked green external peel removed) of each cultivar were diced into $1 \mathrm{~cm}^{3}$ pieces using a knife and mixed thoroughly with a spatula. The flesh and rind were blended separately for $\sim 20 \mathrm{~s}$ using a mini blender (GForce, Hong Kong, China) until a homogeneous consistency was reached. ${ }^{\circ}$ Brix of the watermelon flesh ranged 10.5-11.5, while rinds had ${ }^{\circ}$ Brix of 5.0-5.5. ${ }^{\circ}$ Brix was measured using a pocket refractometer (Atago Co., Ltd., Tokyo, Japan). The samples were stored at $-20^{\circ} \mathrm{C}$ until volatile analysis.

\subsection{Volatile Extraction Using SPME}

Frozen watermelon rind and flesh were thawed to room temperature for $30 \mathrm{~min}$ prior to analysis. A 3 g portion of each sample was placed in $20 \mathrm{~mL}$ glass headspace vials which contained $1 \mathrm{~g}$ of $\mathrm{NaCl}$ (analytical grade, ACROS Organics, Fair Lawn, NJ, USA). The vials were sealed with $18 \mathrm{~mm}$ magnetic screw craps fitted with a PTFE blue silicone septum. A solid-phase microextraction (SPME) fiber coated with divinylbenzene/carboxen/polydimethylsiloxane (DVB/CAR/PDMS, 50/30 $\mu \mathrm{m}$ film thickness, Supelco, Bellefonte, PA, USA) was used in combination with an automated AOC-6000 sampler (Shimadzu, Columbia, MD, USA). The SPME fiber was pre-conditioned at $200{ }^{\circ} \mathrm{C}$ for $3 \mathrm{~min}$. The sample vial was incubated to reach equilibrium at $40{ }^{\circ} \mathrm{C}$ for 15 min within a SPME chamber. Volatile headspace extraction was performed by inserting and holding the SPME fiber in the sample vial headspace to a depth of $22 \mathrm{~mm}$ for $20 \mathrm{~min}$, maintaining agitation at 
$250 \mathrm{rpm}$. Extracted volatiles by the SPME fiber were thermally desorbed for $3 \mathrm{~min}$ into a splitless GC injection port set to $250{ }^{\circ} \mathrm{C}$ [31].

\subsection{GC-MS Analysis}

Volatile separation and identification were performed with gas chromatography-mass spectrometry (GC-MS), which was a 2010 Plus GC coupled with a QP2020 MS (Shimadzu, Columbia, MD, USA). The GC-MS was coupled with an auto sampler (AOC-6000). A ZB-Wax column $(30.0 \mathrm{~m} \times 0.25 \mathrm{~mm}$ i.d. $\times 0.25 \mu \mathrm{m}$ film thickness, Phenomenex, Torrance, CA, USA) was used to separate the compounds. A helium carrier gas flowed at $1.0 \mathrm{~mL} / \mathrm{min}$. The oven temperature was held for $1 \mathrm{~min}$ at $40{ }^{\circ} \mathrm{C}$, then ramped up at $5{ }^{\circ} \mathrm{C} / \mathrm{min}$ to $230{ }^{\circ} \mathrm{C}$ and held for $5 \mathrm{~min}$. The mass spectrometer used an ionization source at $200^{\circ} \mathrm{C}$, an interface temperature of $230^{\circ} \mathrm{C}$, and a scan model over a fragment range of $\mathrm{m} / z$ 35-350 [31].

A 1:100 dilution of a purchased straight-chain C6-C26 alkane mixture (Supelco, Bellefonte, PA, USA) was ran through the same SPME-GC-MS using 1 min equilibration and 5 min extraction times for SPME. The linear retention indices (LRI) of the identified compounds were calculated based on the retention times of these alkanes. Compounds were tentatively identified by matching their ion spectra chromatogram with those from the National Institute of Standards and Technology (NIST) library and confirmed by comparing their LRI with values from literature, especially from NIST Chemistry Webbook, SRC 69. Relative compound quantities (percent of peak area) were calculated based on the ion peak areas integrated by the GC-MS program.

\subsection{Statistical Analysis}

Each sample was prepared and analyzed in triplicate with three watermelon fruits per cultivar. Volatile compounds in samples were compared by measurements of peak areas (abundance) and relative peak areas (percentages). Two-way analysis of variance (ANOVA) was conducted to understand if volatile abundance was dependent on cultivar, fruit part (flesh vs. rind), or both. One-way ANOVA and Tukey's HSD (honestly significant difference) test were conducted within cultivar samples of rind and flesh to determine significant differences in their volatile abundance. Principal component analysis (PCA) was conducted to visualize sample differences and volatiles associated with each sample, filtering out observations with squared cosines sum $\left(\cos ^{2}\right)<0.5$. Heat map cluster analysis was conducted to visualize patterns of volatile abundance across the eight samples. Volatiles with an interquartile range below 0.01 were removed from heat map analysis due to their low variability. All ANOVA was conducted using SPSS version 25 (IMB SPSS, Armonk, NY, USA), while PCA and heat map cluster analysis were conducted using XLSTAT version 2019 (Addinsoft, New York, NY, USA). All tests assumed a significance level of $\alpha \leq 0.05$.

\section{Results}

\subsection{Watermelon Rind and Flesh Total and Grouped Volatiles}

As shown in Figure 1 for the total volatiles identified in the rinds and flesh of four watermelon cultivars, the total volatile abundance (peak area) in the rinds was only $28-58 \%$ of the volatile abundance recorded in the corresponding flesh samples. Captivation had the highest volatile abundance in its rind and flesh, while Fascination had the lowest amount for both. 


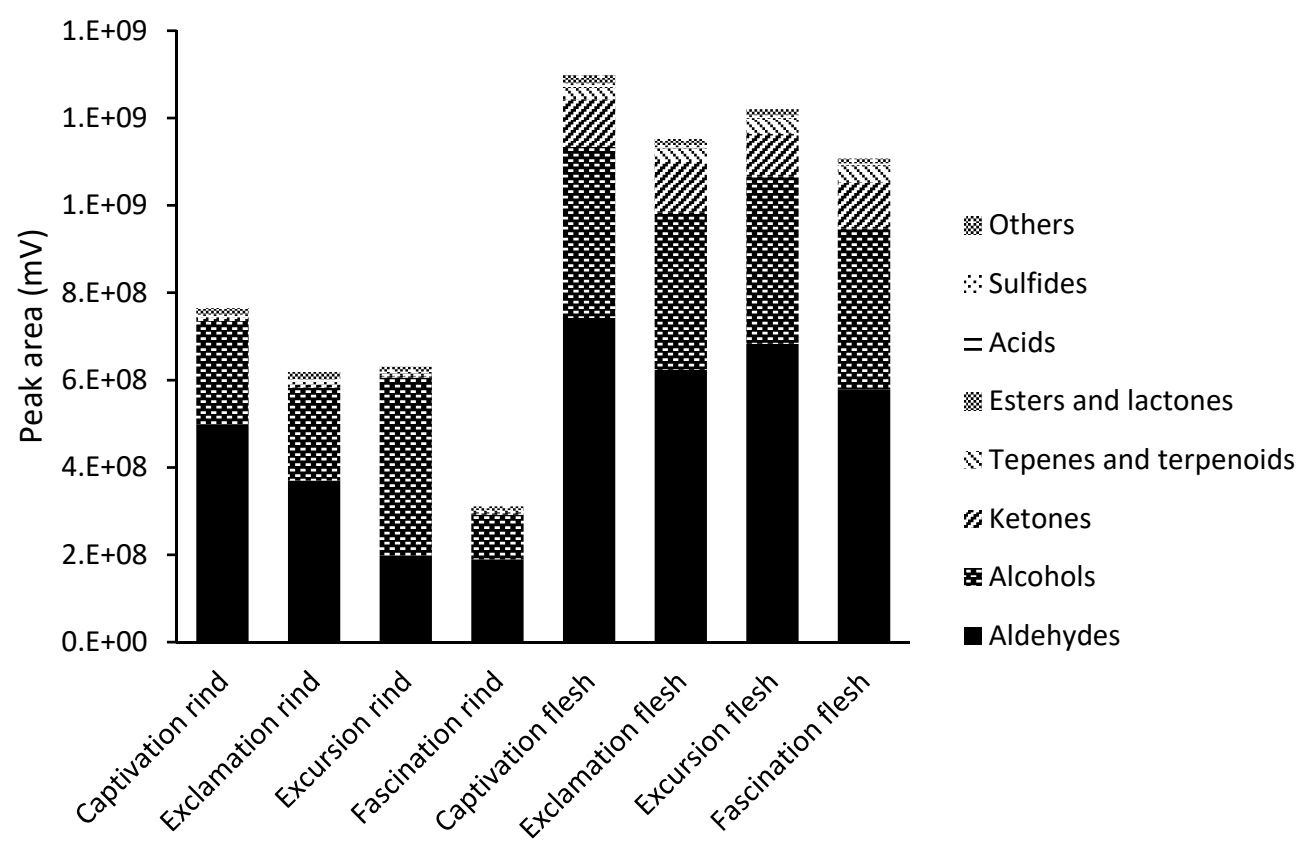

Figure 1. Group of volatiles (sum of peak areas, millivolts $(\mathrm{mV})$ ) in the rinds and fleshes of four watermelon cultivars of Captivation, Exclamation, Excursion, and Fascination using SPME-GCMS analysis.

Volatiles were classified into nine groups based on their chemical structure, namely, aldehydes, alcohols, ketones, terpenes/terpenoids, esters, lactones, acids, sulfides, and others (mainly, furans and aromatics), as shown in Figure 1. The major volatile groups were aldehydes and alcohols based on numbers and quantity (peak abundance) in both rind and flesh, while ketones were the third most abundant in flesh. A greater number of volatiles were identified in the flesh than rind; the flesh also had greater total volatile intensity (cumulative peak area). The remaining volatile groups (terpenes/terpenoids, esters, lactones, acids, ands sulfides) were minimal for all eight samples.

Watermelon rind primarily consisted of aldehydes (31.4-65.1\% of identifications) and alcohols (31.1-64.7\% of identifications), summing to $94-96 \%$ of total peak areas as shown in Figure 1. The remaining 3.8-6.1\% of volatiles were represented by ketones, terpenes/terpenoids, esters, lactones, acids, sulfurs, furans, and aromatics. Within four rind samples, it presented additional differences in total peak area and presence of volatiles in specific cultivars. Captivation had the greatest abundance (peak area) of volatiles, followed by Excursion, Exclamation, and Fascination. Fascination only had about half the amount compared to the other three cultivars. Interestingly, aldehydes were more abundant in the rinds of Captivation, Exclamation, and Fascination $(65.1 \%, 59.8 \%$, and $60.9 \%$, respectively) compared to the rind of Excursion $(31.4 \%)$, in which alcohols were most abundant $(64.7 \%)$. Captivation, Exclamation, and Fascination, however, only contained $31.1 \%, 34.4 \%$, and $33.0 \%$ alcohols, respectively.

Similarly, aldehyde and alcohols were dominant in watermelon flesh, and were present in similar abundances (peak area) across all cultivars (52.2-57.1\% aldehydes and 30.2-33.3\% alcohols), as shown in Figure 1. The total amount of aldehydes and alcohols was 85.4-7.4\%, while summed aldehydes, alcohols, and ketones accounted for $95.1-95.8 \%$ of the total volatiles. Terpenes/terpenoids, esters, lactones, acids, sulfur-containing compounds, and others represented the remaining $4.2-4.9 \%$ of the total volatiles. Each cultivar had a unique volatile profile. Captivation had the greatest total amount of volatiles, followed by Excursion, Exclamation, and Fascination. 


\subsection{Watermelon Rind Major Volatiles and Cultivar Difference}

A total of 132 volatiles were identified in eight samples (Table 1 ). There were 105 volatiles present in four rind samples, while only 75 volatiles were found in common between all four cultivars of rind. The most dominant (at least $2 \%$ peak area in all samples) were ( $Z$ )-3-nonen1-ol (12.4-23.9\%), (Z,Z)-3,6-nonadien-1-ol (7.6-21.4\%), (E,Z)-2,6-nonadienal (5.6-13.6\%), hexanal (7.4-11.5\%), (E)-2-nonenal (3.3-10.8\%), and acetaldehyde (2.5-4.1\%). They were significantly different $(p \leq 0.05)$ in abundance between rind samples. Excursion had the greatest abundance of ( $Z$ )-3-nonen-1-ol and (Z,Z)-3,6-nonadien-1-ol, while Exclamation and Fascination had the lowest abundance of each, respectively. Captivation had the greatest abundance of (E,Z)-2,6-nonadienal and (E)-2-nonenal, while Excursion had the lowest in both. Exclamation had the greatest abundance of hexanal, Fascination had the greatest abundance of acetaldehyde, and Captivation had the lowest abundance of both.

A number of volatiles were present in all samples, yet only dominant in one particular cultivar while having a low abundance (less than $2 \%$ peak area) in others, $p \leq 0.05$. The rind of Captivation exhibited a high abundance of $(E)$-2-hexenal $(10.6 \%)$ particularly compared to Fascination $(0.9 \%)$, while Fascination was more abundant in nonanal $(12.6 \%)$ compared to Excursion (0.7\%). Fascination rind also contained abundant (Z)-6-nonenal (12.1\%) compared to no detection in Exclamation. Excursion was more abundant in 1-hexanol (4.3\%), (Z)-3-hexen-1-ol (5.7\%), and (Z)-6-nonenol (2.6\%) compared to all remaining three rind samples $(<2 \%)$.

There were 57 volatiles not present in all four cultivars, and eight of those were unique to certain cultivars. Those unique volatiles included $(E)$-2-nonenol (0.06\%), 2-ethylhexanoic acid $(0.02 \%)$, and ethyl cinnamate $(0.01 \%)$ present in Captivation, and ethanol $(0.3 \%)$, 2-heptanone $(0.03 \%)$, and 1-heptanol $(0.2 \%)$ in Excursion.

There were several volatiles found in all of the rind samples that have never been reported in watermelon. The most dominant of those volatiles, which ranged from 0.01 to $0.8 \%$ abundance, included $(E, E)-2,6$-nonadienal $(0.06-0.17 \%)$, methanol $(0.15-0.80 \%)$, dihydromyrcenol $(0.07-0.23 \%)$, and isobornyl acetate $(0.03-0.11 \%)$. The remaining volatiles had an abundance of less than $0.1 \%$ in the samples, including (Z)-3-hepten-1-ol (0.02-0.08\%), 2-octanone $(0.02-0.06 \%)$, 2-undecanone $(0.02-0.07 \%)$, tetrahydrolinalool $(0.02-0.08 \%)$, heptanoic acid (0.04-0.09\%), and dimethyl disulfide (0.01-0.07\%).

\subsection{Watermelon Flesh Major Volatiles and Cultivar Difference}

Among 132 volatiles identified in eight samples, analysis recovered 114 volatile compounds from each of the Captivation, Exclamation, Excursion, and Fascination flesh samples (Table 1). There were 97 volatiles found in common between all cultivars of watermelon flesh, while 17 volatiles only occurred in certain varieties. The dominant volatiles (at least $2 \%$ peak area in all samples) being known watermelon flavor compounds including hexanal (12.7-15.1\%), (E)-2-nonenal (7.4-13.0\%), (Z)-3-nonen-1-ol (8.1-12.3\%), 6-methyl-5-hepten2-one (6.4-8.4\%), (E,Z)-2,6-nonadienal (5.3-9.8\%), (Z)-6-nonenal (2.1-6.6\%), nonanal (4.4$6.0 \%),(Z, Z)-3,6$-nonadien-1-ol (3.4-6.0\%), hexanol (3.6-5.8\%), and (E)-2-octenal (2.2-2.9\%). The abundance of those volatiles differed significantly $(p \leq 0.05)$ between cultivars. Excursion had the lowest abundance of hexanal, 6-methyl-5-hepten-2-one and nonanal, while Exclamation and Captivation had the greatest of those. Fascination had the lowest abundance of (Z)-6-nonenal, (E,Z)-2,6-nonadienal, and (Z,Z)-3,6-nonadien-1-ol, while Excursion had the greatest abundance of the former two. Captivation had the greatest abundance of the latter. Captivation had the lowest abundance of (E)-2-nonenal and (Z)-3-nonen-1-ol, while Fascination had the greatest abundance of those. 
Table 1. Rind and flesh volatiles of Captivation, Exclamation, Excursion, and Fascination watermelon cultivars (\% of peak area).

\begin{tabular}{|c|c|c|c|c|c|c|c|c|c|c|c|c|c|c|c|c|}
\hline \multirow{2}{*}{ V\# } & \multirow{2}{*}{$\begin{array}{l}\text { LRI } \\
\text { Wax }\end{array}$} & \multirow{2}{*}{ Compounds } & \multirow{2}{*}{ CAS\# } & \multicolumn{4}{|c|}{ Watermelon Rind } & \multirow{2}{*}{$p$-Value } & \multicolumn{4}{|c|}{ Watermelon Flesh } & \multirow{2}{*}{$p$-Value } & \multicolumn{3}{|c|}{ F-Value } \\
\hline & & & & Captivation & Exclamation & Excursion & Fascination & & Captivation & Exclamation & Excursion & Fascination & & Cultivar (C) & Fruit Part (P) & $\mathbf{C} \times \mathbf{P}$ \\
\hline & & Aldehydes (33) & & & & & & & & & & & & & & \\
\hline V1 & 636 & Acetaldehyde & $75-07-0$ & $2.51 \mathrm{a}$ & $3.20 \mathrm{ab}$ & 3.71ab & $4.13 \mathrm{~b}$ & 0.048 & $1.46 \mathrm{a}$ & $2.04 \mathrm{ab}$ & $2.59 \mathrm{~b}$ & $2.54 \mathrm{~b}$ & 0.021 & 8.85 & 35.35 & 0.38 \\
\hline V2 & 697 & Propanal & & $1.35 \mathrm{a}$ & & & - & 0.905 & $0.70 \mathrm{a}$ & $0.65 a$ & $0.71 \mathrm{a}$ & $0.59 a$ & 0.650 & 0.21 & 29.23 & 0.14 \\
\hline V3 & 841 & 2-Methylbutanal & $96-17-3$ & $0.02 \mathrm{a}$ & $0.03 a$ & $0.02 \mathrm{a}$ & $0.03 a$ & 0.697 & $0.03 \mathrm{ab}$ & $0.03 \mathrm{~b}$ & $0.03 \mathrm{ab}$ & $0.02 a$ & 0.023 & 0.68 & 1.13 & 0.78 \\
\hline $\mathrm{V} 4$ & 843 & 3-Methylbutanal & $590-86-3$ & $0.02 \mathrm{a}$ & $0.02 \mathrm{a}$ & $0.02 \mathrm{a}$ & $0.03 a$ & 0.652 & $0.03 \mathrm{~b}$ & $0.03 \mathrm{~b}$ & $0.03 \mathrm{ab}$ & $0.02 \mathrm{a}$ & 0.015 & 0.17 & 0.81 & 1.59 \\
\hline V5 & 880 & Pentanal & $110-62-3$ & $0.31 \mathrm{ab}$ & $0.88 \mathrm{c}$ & $0.53 \mathrm{~b}$ & $0.21 \mathrm{a}$ & $<0.001$ & $0.62 \mathrm{~b}$ & $0.50 \mathrm{ab}$ & $0.45 \mathrm{ab}$ & $0.39 a$ & 0.016 & 23.67 & 0.05 & 21.93 \\
\hline V6 & 1031 & (E)-2-Butenal & $4170-30-3$ & & & & & & $0.07 \mathrm{a}$ & $0.09 \mathrm{a}$ & $0.14 \mathrm{~b}$ & & 0.011 & & & \\
\hline V7 & 1055 & 2-Ethyl-3-methylbutanal & $26254-92-2$ & $0.01 \mathrm{a}$ & $0.02 \mathrm{a}$ & $0.01 \mathrm{a}$ & $0.01 \mathrm{a}$ & 0.383 & $0.09 \mathrm{~b}$ & $0.06 \mathrm{ab}$ & $0.06 \mathrm{ab}$ & $0.04 a$ & 0.025 & 4.10 & 120.8 & 5.55 \\
\hline V8 & 1075 & Hexanal & $66-25-1$ & $7.42 \mathrm{a}$ & $11.53 \mathrm{~b}$ & $8.39 a$ & $8.54 a$ & 0.002 & $15.07 \mathrm{~b}$ & $15.12 \mathrm{~b}$ & $12.70 \mathrm{a}$ & $14.83 \mathrm{~b}$ & 0.013 & 12.67 & 267.8 & 7.81 \\
\hline V9 & 1121 & (E)-2-Pentenal & $1576-87-0$ & $0.17 \mathrm{a}$ & $0.18 \mathrm{a}$ & $0.18 \mathrm{a}$ & $0.23 \mathrm{a}$ & 0.158 & $0.53 \mathrm{c}$ & $0.36 \mathrm{~b}$ & $0.48 \mathrm{c}$ & $0.25 a$ & $<0.001$ & 15.69 & 281.3 & 35.65 \\
\hline V10 & 1130 & (E)-3-Hexenal & 69112-21-6 & $0.11 \mathrm{a}$ & $0.10 \mathrm{a}$ & $0.09 \mathrm{a}$ & $0.11 \mathrm{a}$ & 0.682 & $0.03 a$ & - & $0.04 a$ & - & 0.062 & 0.10 & 42.97 & 2.49 \\
\hline V12 & 1169 & 2-Methyl-2-pentenal & $28467-88-1$ & & - & - & - & - & $0.01 \mathrm{a}$ & $0.01 \mathrm{a}$ & $0.01 \mathrm{a}$ & $0.005 a$ & 0.291 & - & - & - \\
\hline V13 & 1176 & Heptanal & $111-71-7$ & $0.06 \mathrm{ab}$ & $0.08 \mathrm{~b}$ & $0.03 a$ & $0.12 \mathrm{c}$ & $<0.001$ & $0.65 \mathrm{~b}$ & $0.51 \mathrm{ab}$ & $0.46 a b$ & $0.42 \mathrm{a}$ & 0.051 & 3.40 & 292.1 & 5.72 \\
\hline V14 & 1191 & 3-Methyl-2-butenal & $107-86-8$ & & & & - & & $0.01 \mathrm{a}$ & $0.02 \mathrm{~b}$ & & $0.02 \mathrm{~b}$ & 0.004 & & - & \\
\hline V15 & 1194 & (Z)-2-Hexenal & $16635-54-4$ & $0.24 \mathrm{~b}$ & $0.20 \mathrm{ab}$ & $0.17 a$ & - & 0.003 & $0.02 a$ & $0.03 a$ & $0.05 \mathrm{a}$ & $0.02 \mathrm{a}$ & 0.358 & 6.47 & 47.7 & 24.8 \\
\hline V16 & 1204 & 3,3-Dimethylhexanal & $55320-57-5$ & & & & - & & $0.29 \mathrm{a}$ & $0.25 a$ & $0.17 \mathrm{a}$ & $0.22 \mathrm{a}$ & 0.237 & & & \\
\hline V17 & 1210 & (E)-2-Hexenal & $6728-26-3$ & $10.57 d$ & $6.91 \mathrm{c}$ & $5.05 \mathrm{~b}$ & $0.93 a$ & $<0.001$ & $1.41 \mathrm{~d}$ & $1.00 \mathrm{c}$ & $0.80 \mathrm{~b}$ & $0.49 a$ & $<0.001$ & 539.0 & 2708.4 & 367.7 \\
\hline V18 & 1281 & Octanal & $124-13-0$ & $0.12 \mathrm{~b}$ & $0.31 \mathrm{c}$ & $0.04 a$ & $0.46 \mathrm{~d}$ & $<0.001$ & $1.27 \mathrm{~b}$ & $0.92 \mathrm{ab}$ & $0.79 a$ & $0.75 a$ & 0.017 & 5.92 & 209.7 & 14.49 \\
\hline V19 & 1315 & (E)-2-Heptenal & $57266-86-1$ & $0.18 \mathrm{a}$ & $0.21 \mathrm{a}$ & $0.11 \mathrm{a}$ & $0.12 \mathrm{a}$ & 0.098 & $2.00 \mathrm{a}$ & $1.68 \mathrm{a}$ & $1.52 \mathrm{a}$ & $1.61 \mathrm{a}$ & 0.270 & 2.00 & 316.8 & 1.22 \\
\hline V20 & 1388 & Nonanal & $124-19-6$ & $6.95 \mathrm{~b}$ & $7.07 \mathrm{~b}$ & $0.67 \mathrm{a}$ & $12.62 \mathrm{c}$ & $<0.001$ & $5.97 \mathrm{~b}$ & $5.84 \mathrm{~b}$ & $5.15 \mathrm{~b}$ & $4.40 \mathrm{a}$ & $<0.001$ & 74.33 & 51.25 & 23.49 \\
\hline V21 & 1422 & (E)-2-Octenal & $2548-87-0$ & $0.38 \mathrm{~b}$ & $0.37 \mathrm{~b}$ & $0.21 \mathrm{a}$ & $0.44 \mathrm{~b}$ & 0.001 & $2.34 \mathrm{ab}$ & $2.15 a$ & $2.24 \mathrm{ab}$ & $2.91 \mathrm{~b}$ & 0.038 & 6.39 & 657.0 & 3.30 \\
\hline V22 & 1435 & (E)-6-Nonenal & $2277-20-5$ & $0.08 \mathrm{~b}$ & - & $0.03 a$ & $0.09 \mathrm{~b}$ & $<0.001$ & $0.16 \mathrm{a}$ & $0.19 \mathrm{a}$ & $0.15 a$ & $0.13 a$ & 0.646 & 46.25 & 63.07 & 83.06 \\
\hline V23 & 1445 & (Z)-6-Nonenal & $2277-19-2$ & $8.77 \mathrm{~b}$ & $12.08 \mathrm{c}$ & $1.02 \mathrm{a}$ & $12.07 \mathrm{c}$ & $<0.001$ & $6.62 \mathrm{c}$ & $4.41 \mathrm{~b}$ & $3.67 \mathrm{~b}$ & $2.07 \mathrm{a}$ & $<0.001$ & 1692.4 & 1576.5 & 1667.1 \\
\hline V24 & 1493 & Decanal & $112-31-2$ & & $0.04 \mathrm{a}$ & $0.05 a$ & $0.17 \mathrm{~b}$ & 0.004 & $0.10 \mathrm{ab}$ & $0.09 a$ & $0.11 a b$ & $0.14 \mathrm{~b}$ & 0.016 & 17.16 & 4.18 & 7.44 \\
\hline V25 & 1499 & (Z)-2-Nonenal & $18829-56-6$ & $0.29 \mathrm{~b}$ & $0.14 a$ & $0.12 a$ & $0.36 \mathrm{~b}$ & $<0.001$ & $0.30 \mathrm{a}$ & $0.31 \mathrm{a}$ & $0.47 a$ & $0.46 a$ & 0.180 & 4.99 & 22.75 & 4.64 \\
\hline V28 & 1567 & $(E, E)$-2,6-Nonadienal & $17587-33-6$ & $0.17 \mathrm{c}$ & $0.10 \mathrm{~b}$ & $0.06 \mathrm{a}$ & $0.16 \mathrm{c}$ & $<0.001$ & $0.19 \mathrm{c}$ & $0.15 \mathrm{~b}$ & $0.19 \mathrm{c}$ & $0.06 \mathrm{a}$ & $<0.001$ & 40.43 & 25.52 & 96.07 \\
\hline V29 & 1581 & $(E, Z)-2,6-$ Nonadienal & $557-48-2$ & $13.63 \mathrm{c}$ & $9.34 \mathrm{~b}$ & $5.64 a$ & $9.88 \mathrm{~b}$ & $<0.001$ & $8.12 \mathrm{~b}$ & $6.91 \mathrm{~b}$ & $9.77 \mathrm{c}$ & $5.30 \mathrm{a}$ & $<0.001$ & 32.12 & 59.37 & 62.98 \\
\hline V30 & 1637 & (E)-2-Decenal & $3913-81-3$ & $0.02 \mathrm{a}$ & $0.01 \mathrm{a}$ & $0.01 \mathrm{a}$ & $0.01 \mathrm{a}$ & 0.432 & $0.33 a$ & $0.30 \mathrm{a}$ & $0.39 \mathrm{a}$ & $0.40 \mathrm{a}$ & 0.365 & 1.11 & 249.6 & 1.32 \\
\hline V31 & 1803 & $(E, E)$-2,4-Decadienal & $25152-84-5$ & $0.01 \mathrm{a}$ & $0.01 \mathrm{a}$ & $0.01 \mathrm{a}$ & $0.01 \mathrm{a}$ & 0.194 & $0.14 \mathrm{a}$ & $0.10 \mathrm{a}$ & $0.12 \mathrm{a}$ & $0.11 \mathrm{a}$ & 0.453 & 0.75 & 102.9 & 0.88 \\
\hline V32 & 1824 & 4-Oxononanal & $74327-29-0$ & $0.20 \mathrm{~b}$ & $0.31 \mathrm{c}$ & $0.07 \mathrm{a}$ & $0.42 \mathrm{~d}$ & $<0.001$ & $0.81 \mathrm{a}$ & $0.78 \mathrm{a}$ & $0.75 \mathrm{a}$ & $0.88 \mathrm{a}$ & 0.066 & 39.07 & 1208.6 & 12.31 \\
\hline V33 & 2005 & (E)-Cinnamaldehyde & $14371-10-9$ & $0.01 \mathrm{a}$ & $0.01 \mathrm{a}$ & $0.01 \mathrm{ab}$ & $0.01 \mathrm{~b}$ & 0.033 & $0.01 \mathrm{a}$ & $0.01 \mathrm{ab}$ & $0.01 \mathrm{~b}$ & $0.01 \mathrm{ab}$ & 0.036 & 6.36 & 10.77 & 3.17 \\
\hline & & Alcohols (33) & & & & & & & & & & & & & & \\
\hline $\begin{array}{l}\text { V34 } \\
\text { V35 }\end{array}$ & $\begin{array}{l}832 \\
851\end{array}$ & $\begin{array}{l}\text { Methanol } \\
\text { Ethanol }\end{array}$ & $\begin{array}{l}67-56-1 \\
64-17-5\end{array}$ & $0.17 a$ & $0.15 a$ & $\begin{array}{l}0.16 \mathrm{a} \\
0.27\end{array}$ & $0.80 \mathrm{~b}$ & $\begin{array}{l}<0.001 \\
-\end{array}$ & $\begin{array}{l}0.23 a \\
1.28 a\end{array}$ & $\begin{array}{l}0.22 \mathrm{a} \\
3.44 \mathrm{bc}\end{array}$ & $\begin{array}{l}0.24 a \\
3.96 c\end{array}$ & $\begin{array}{l}0.22 \mathrm{a} \\
3.27 \mathrm{~b}\end{array}$ & $\begin{array}{l}0.830 \\
<0.001\end{array}$ & $\begin{array}{l}631.4 \\
97.53\end{array}$ & 220.1 & 657.2 \\
\hline $\begin{array}{l}\text { V35 } \\
\text { V36 }\end{array}$ & $\begin{array}{l}851 \\
1103\end{array}$ & $\begin{array}{l}\text { Ethanol } \\
\text { 3-Pentanol }\end{array}$ & $\begin{array}{l}64-17-5 \\
584-02-1\end{array}$ & - & $0.01 \mathrm{a}$ & $0.02 \mathrm{a}$ & $0.02 \mathrm{a}$ & 0.069 & $1.28 \mathrm{a}$ & $\begin{array}{l}3.44 \mathrm{DC} \\
0.01\end{array}$ & $3.96 \mathrm{c}$ & $\begin{array}{l}3.27 \mathrm{~b} \\
-\end{array}$ & $\begin{array}{l}<0.001 \\
-\end{array}$ & $\begin{array}{l}97.53 \\
5.47\end{array}$ & $\begin{array}{l}479.4 \\
21.88\end{array}$ & - \\
\hline V37 & 1136 & 1-Butanol & $71-36-3$ & & & & & & $0.04 \mathrm{a}$ & $0.07 a b$ & $0.10 \mathrm{~b}$ & $0.09 \mathrm{~b}$ & 0.016 & & & \\
\hline V38 & 1149 & 2-Methyl-3-pentanol & $623-37-0$ & $0.03 a$ & $0.03 a$ & $0.05 \mathrm{~b}$ & $0.09 \mathrm{c}$ & 0.016 & $0.02 a$ & $0.03 a$ & $0.03 a$ & $0.03 a$ & 0.275 & 20.26 & 50.31 & 13.47 \\
\hline V39 & 1152 & 1-Penten-3-ol & $616-25-1$ & $0.25 a$ & $0.43 \mathrm{~b}$ & $0.46 \mathrm{~b}$ & $0.36 \mathrm{ab}$ & 0.014 & $0.22 \mathrm{~b}$ & $0.19 \mathrm{~b}$ & $0.19 \mathrm{~b}$ & $0.12 \mathrm{a}$ & 0.003 & 6.31 & 105.8 & 8.21 \\
\hline V40 & 1199 & 2-Methylbutanol & $137-32-6$ & & $0.01 \mathrm{a}$ & $0.02 \mathrm{a}$ & $0.03 \mathrm{a}$ & 0.132 & $0.11 \mathrm{a}$ & $0.27 \mathrm{~b}$ & $0.21 \mathrm{~b}$ & $0.08 \mathrm{a}$ & $<0.001$ & 35.04 & 374.2 & 53.85 \\
\hline V41 & 1242 & 1-Pentanol & $71-41-0$ & $0.43 a$ & $1.03 c$ & $0.73 \mathrm{~b}$ & $0.33 \mathrm{a}$ & $<0.001$ & $1.35 a$ & $1.39 a$ & - & $1.27 a$ & 0.530 & 18.07 & 223.5 & 15.19 \\
\hline V42 & 1314 & (Z)-2-Penten-1-ol & $33467-76-4$ & $0.08 \mathrm{a}$ & $0.19 \mathrm{~b}$ & $0.15 \mathrm{ab}$ & $0.13 \mathrm{ab}$ & 0.05 & & & & & & & & \\
\hline V43 & 1346 & 1-Hexanol & $111-27-3$ & $1.42 \mathrm{~b}$ & $1.73 \mathrm{~b}$ & $4.29 \mathrm{c}$ & $0.60 \mathrm{a}$ & $<0.001$ & $4.19 \mathrm{a}$ & $5.48 \mathrm{~b}$ & $3.61 \mathrm{a}$ & $5.79 \mathrm{~b}$ & $<0.001$ & 39.20 & 355.0 & 456.3 \\
\hline V44 & 1356 & (E)-3-Hexen-1-ol & $928-97-2$ & $0.02 \mathrm{~b}$ & $0.02 \mathrm{~b}$ & $0.08 \mathrm{c}$ & $0.01 \mathrm{a}$ & $<0.001$ & $0.03 \mathrm{~d}$ & $0.02 \mathrm{c}$ & $0.02 \mathrm{~b}$ & $0.01 \mathrm{a}$ & 0.055 & 13.30 & 0.15 & 0.05 \\
\hline V45 & 1376 & (Z)-3-Hexen-1-ol & 928-96-1 & $1.74 \mathrm{~b}$ & $1.84 \mathrm{~b}$ & $5.66 c$ & $0.21 \mathrm{a}$ & $<0.001$ & $0.71 \mathrm{c}$ & $0.80 \mathrm{c}$ & $0.58 \mathrm{~b}$ & $0.38 a$ & $<0.001$ & 311.0 & 991.9 & 457.9 \\
\hline V46 & 1399 & (E)-2-Hexen-1-ol & 928-95-0 & $0.05 a$ & & $0.09 \mathrm{~b}$ & & 0.045 & & & & & & & & \\
\hline V47 & 1449 & 1-Heptanol & $111-70-6$ & & & 0.17 & & & $1.12 \mathrm{~b}$ & $0.90 \mathrm{a}$ & $0.83 a$ & $0.94 a$ & $<0.001$ & & & \\
\hline V48 & 1458 & 6-Methylhept-5-en-2-ol & $1569-60-4$ & $0.03 a$ & $0.08 \mathrm{bc}$ & $0.09 \mathrm{c}$ & $0.06 \mathrm{~b}$ & $<0.001$ & $1.48 \mathrm{ab}$ & $1.53 \mathrm{ab}$ & $1.68 \mathrm{~b}$ & $1.25 \mathrm{a}$ & 0.060 & 4.31 & 956.5 & 3.30 \\
\hline $\mathrm{V} 49$ & 1472 & 2,4-Dimethyl-1-hepten-4-ol & 19549-94-1 & & & & & & $0.06 a$ & $0.04 a$ & $0.03 a$ & $0.02 \mathrm{a}$ & 0.266 & & & \\
\hline V50 & 1477 & (Z)-3-Hepten-1-ol & $2108-05-6$ & $0.02 \mathrm{a}$ & $0.02 \mathrm{a}$ & $0.08 \mathrm{~b}$ & $0.02 \mathrm{a}$ & $<0.001$ & $0.03 a$ & $0.03 a$ & $0.02 \mathrm{a}$ & $0.01 \mathrm{a}$ & 0.363 & 10.48 & 6.50 & 8.07 \\
\hline V51 & 1485 & 2-Éthylhexanol & $104-76-7$ & $0.36 \mathrm{a}$ & $0.62 \mathrm{~b}$ & $0.49 \mathrm{ab}$ & $1.12 \mathrm{c}$ & $<0.001$ & $0.63 \mathrm{~b}$ & $0.37 \mathrm{a}$ & $0.37 \mathrm{a}$ & $0.27 \mathrm{a}$ & $<0.001$ & 15.02 & 63.68 & 59.92 \\
\hline V52 & 1553 & 1-Octanol & $111-87-5$ & $0.65 a$ & $0.26 a$ & $0.45 a$ & $0.70 \mathrm{a}$ & 0.130 & $1.46 \mathrm{~b}$ & $1.07 \mathrm{a}$ & $1.22 \mathrm{ab}$ & $1.39 \mathrm{~b}$ & 0.004 & 7.04 & 112.3 & 0.23 \\
\hline
\end{tabular}


Table 1. Cont.

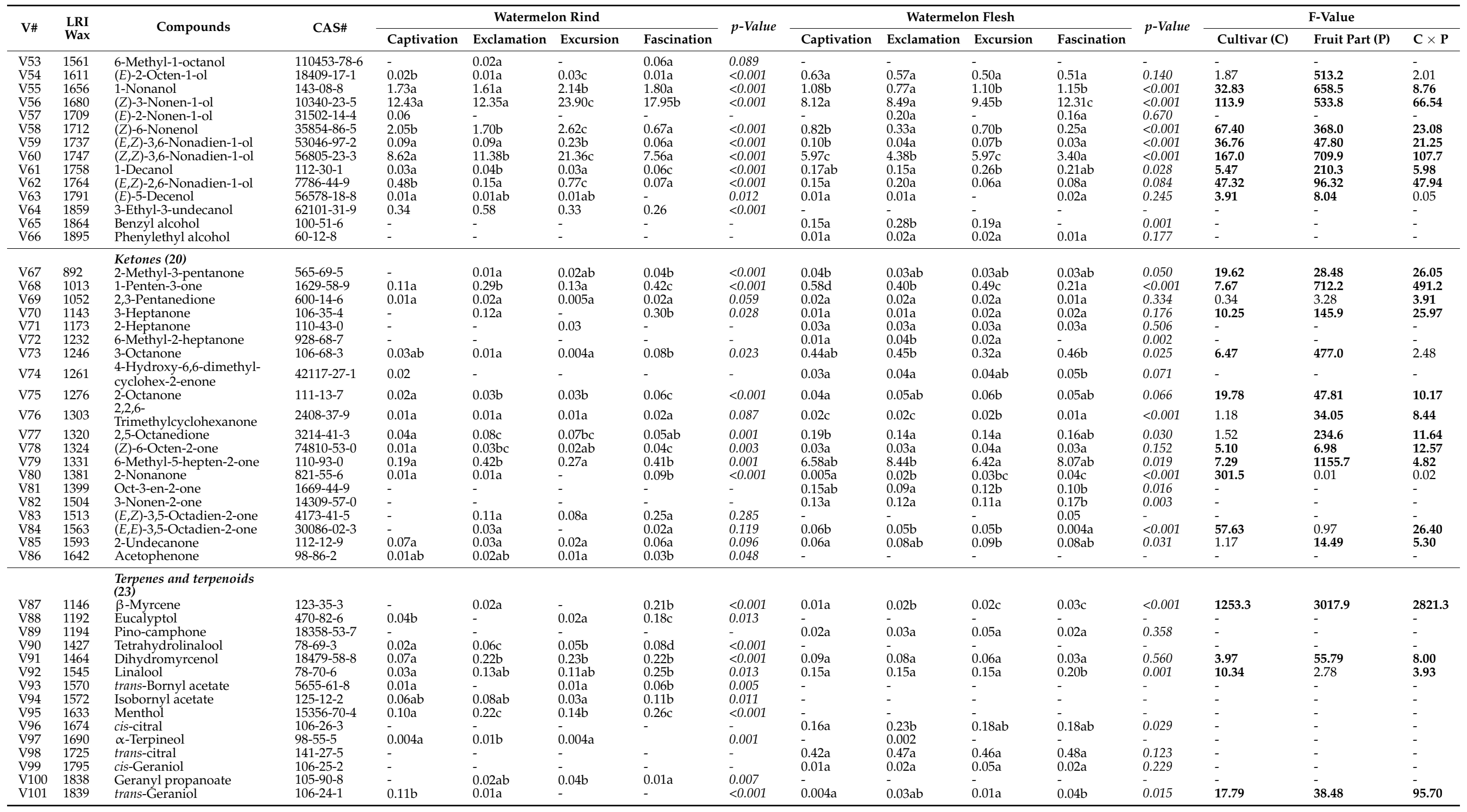


Table 1. Cont.

\begin{tabular}{|c|c|c|c|c|c|c|c|c|c|c|c|c|c|c|c|c|}
\hline \multirow{2}{*}{ V\# } & \multirow{2}{*}{$\begin{array}{l}\text { LRI } \\
\text { Wax }\end{array}$} & \multirow{2}{*}{ Compounds } & \multirow{2}{*}{ CAS\# } & \multicolumn{4}{|c|}{ Watermelon Rind } & \multirow{2}{*}{$p$-Value } & \multicolumn{4}{|c|}{ Watermelon Flesh } & \multirow{2}{*}{$p$-Value } & \multicolumn{3}{|c|}{ F-Value } \\
\hline & & & & Captivation & Exclamation & Excursion & Fascination & & Captivation & Exclamation & Excursion & Fascination & & Cultivar (C) & Fruit Part (P) & $\mathbf{C} \times \mathbf{P}$ \\
\hline V102 & 1846 & trans-Geranylacetone & $3796-70-1$ & $0.06 \mathrm{a}$ & $0.08 \mathrm{~b}$ & $0.08 \mathrm{ab}$ & $0.14 \mathrm{c}$ & $<0.001$ & $0.96 \mathrm{a}$ & $1.46 \mathrm{~b}$ & $1.64 \mathrm{~b}$ & $2.19 \mathrm{c}$ & $<0.001$ & 85.78 & 2509.3 & 66.72 \\
\hline V103 & 1917 & $\beta$-ionone & $79-77-6$ & $0.03 a$ & $0.09 \mathrm{c}$ & $0.05 \mathrm{~b}$ & $-.14 \mathrm{c}$ & $<0.001$ & $0.03 a$ & $0.06 \mathrm{bc}$ & $0.06 \mathrm{~b}$ & $0.08 \mathrm{c}$ & $<0.001$ & 71.42 & 0.62 & 9.74 \\
\hline V104 & 1938 & $\begin{array}{l}\text { 6,10-Dimethyl-5,9- } \\
\text { undecadien-2-o }\end{array}$ & $53837-34-6$ & - & - & - & - & - & $0.03 a$ & $0.02 \mathrm{a}$ & $0.02 \mathrm{a}$ & $0.03 a$ & 0.061 & - & - & - \\
\hline V106 & 2010 & (E,Z)-Psuedoionone & $13927-47-4$ & - & - & - & - & - & $0.01 \mathrm{a}$ & $0.01 \mathrm{a}$ & $0.02 \mathrm{a}$ & $0.01 \mathrm{a}$ & 0.124 & - & - & - \\
\hline V107 & 2087 & $(E, E)$-Psuedoionone & $3548-78-5$ & - & - & - & - & - & $0.01 \mathrm{a}$ & $0.01 \mathrm{a}$ & $0.02 \mathrm{ab}$ & $0.01 \mathrm{a}$ & 0.004 & - & - & - \\
\hline V108 & 2241 & Dihydroactinidiolide & $\begin{array}{l}15356-74-8 \\
117-52-8\end{array}$ & - & - & - & - & - & $\begin{array}{l}0.01 \mathrm{a} \\
0.01 \mathrm{a}\end{array}$ & $\begin{array}{l}0.01 \mathrm{ab} \\
0.02 \mathrm{ab}\end{array}$ & $\begin{array}{l}0.01 \mathrm{~b} \\
0.02 \mathrm{~b}\end{array}$ & $\begin{array}{l}0.01 \mathrm{ab} \\
0.03 \mathrm{c}\end{array}$ & 0.024 & - & - & - \\
\hline VIU9 & 2273 & $\begin{array}{l}\text { Farnesyl acetone } \\
\text { Esters and lactones (9) }\end{array}$ & $1117-52-8$ & & - & - & & & & & & & & & & \\
\hline V110 & 829 & Ethyl Acetate & $141-78-6$ & - & $0.05 a$ & - & $0.87 \mathrm{~b}$ & $<0.001$ & - & - & - & & & - & - & \\
\hline V112 & 1265 & Hexyl acetate & $142-92-7$ & $0.005 a$ & $0.01 a$ & $0.01 \mathrm{a}$ & $0.07 \mathrm{~b}$ & $<0.001$ & $0.01 \mathrm{a}$ & $0.01 \mathrm{a}$ & $0.01 \mathrm{a}$ & $0.01 \mathrm{a}$ & 0.214 & 79.86 & 92.03 & 105.2 \\
\hline V113 & 1622 & 3-6-Nonadien-1-yl acetate & 76649-26-8 & - & - & - & - & - & $0.01 \mathrm{a}$ & & $0.02 \mathrm{a}$ & & 0.175 & - & - & - \\
\hline V114 & 1629 & E-2-hexenyl hexanoate & $3050-69-9$ & - & - & - & - & - & $0.01 \mathrm{a}$ & $0.02 \mathrm{a}$ & - & $0.02 \mathrm{a}$ & 0.200 & - & - & - \\
\hline V115 & 1858 & 2-Ethyl-3-hydroxyhexyl & $74367-31-0$ & - & - & - & - & - & 0.14 & 0.08 & 0.06 & 0.06 & $<0.001$ & - & - & - \\
\hline V116 & 2083 & $\begin{array}{l}\text { 2-methylpropanoate } \\
\text { Ethyl cinnamate }\end{array}$ & $103-36-6$ & 0.01 & & & & & $0.002 \mathrm{a}$ & & & $0.002 \mathrm{a}$ & 0.305 & - & & \\
\hline V117 & 1996 & $\gamma$-Nonalactone & $104-61-0$ & $0.01 \mathrm{a}$ & $0.01 \mathrm{bc}$ & $0.01 \mathrm{ab}$ & $0.02 \mathrm{c}$ & 0.001 & $0.03 a$ & $0.05 \mathrm{~b}$ & $0.05 \mathrm{~b}$ & $0.05 \mathrm{~b}$ & 0.010 & 12.82 & 510.5 & 3.56 \\
\hline V118 & 2103 & lactone of cis-Jasmone & $70851-61-5$ & & & & & & $0.003 \mathrm{a}$ & $0.003 \mathrm{ab}$ & $0.005 \mathrm{bc}$ & $0.01 \mathrm{c}$ & 0.001 & & & \\
\hline & & Acids (5) & & & & & & & & & & & & & & \\
\hline V119 & 1851 & Hexanoic acid & $142-62-1$ & $0.14 \mathrm{~b}$ & $0.05 a$ & $0.08 \mathrm{ab}$ & $0.05 a$ & 0.003 & $0.20 \mathrm{~b}$ & $0.07 \mathrm{a}$ & $0.09 \mathrm{a}$ & $0.03 a$ & 0.001 & 32.56 & 2.69 & 1.86 \\
\hline V120 & 1942 & 2-Ethylhexanoic acid & $149-57-5$ & 0.02 & & & & & - & $0.01 \mathrm{a}$ & & $0.01 \mathrm{a}$ & 0.952 & & & \\
\hline $\begin{array}{l}\text { V121 } \\
\text { V122 }\end{array}$ & $\begin{array}{l}1947 \\
2039\end{array}$ & $\begin{array}{l}\text { Heptanoic acid } \\
\text { Octanoic acid }\end{array}$ & $\begin{array}{l}112-05-0 \\
124-07-2\end{array}$ & $\begin{array}{l}0.09 \mathrm{a} \\
0.06 \mathrm{~b}\end{array}$ & $\begin{array}{l}0.04 \mathrm{a} \\
0.01 \mathrm{a}\end{array}$ & $\begin{array}{l}0.05 \mathrm{a} \\
0.01 \mathrm{a}\end{array}$ & $0.05 a$ & $\begin{array}{l}0.147 \\
<0.001\end{array}$ & $0.04 \mathrm{~b}$ & $0.02 \mathrm{a}$ & $0.03 a$ & $0.02 \mathrm{a}$ & 0.001 & 4.90 & 22.16 & 0.75 \\
\hline V124 & 624 & Methanethiol & 74-93-1 & $0.39 \mathrm{a}$ & $0.62 \mathrm{~b}$ & $0.54 \mathrm{ab}$ & - & 0.039 & $0.370 \mathrm{a}$ & $0.417 \mathrm{a}$ & $0.377 \mathrm{a}$ & $0.243 a$ & 0.159 & 4.83 & 10.43 & 1.78 \\
\hline V125 & 1061 & Dimethyl disulfide & $624-92-0$ & $0.01 \mathrm{a}$ & $0.07 \mathrm{~b}$ & $0.02 \mathrm{a}$ & $0.05 \mathrm{~b}$ & $<0.001$ & $0.02 a$ & $0.02 \mathrm{a}$ & $0.03 a$ & $0.02 \mathrm{a}$ & 0.358 & 33.20 & 14.98 & 18.43 \\
\hline V126 & 1930 & Benzothiazole & $95-16-9$ & $0.01 \mathrm{a}$ & $0.02 \mathrm{bc}$ & $0.01 \mathrm{ab}$ & $0.03 c$ & 0.003 & - & - & - & - & - & - & - & - \\
\hline & & Others (6) & & & & & & & & & & & & & & \\
\hline V127 & 864 & 2-Ethylfuran & 3208-16-0 & $0.07 \mathrm{a}$ & $0.16 \mathrm{~b}$ & $0.14 \mathrm{~b}$ & $-i 14$ & 0.039 & $0.18 \mathrm{c}$ & $0.06 \mathrm{~b}$ & $0.05 \mathrm{ab}$ & $0.03 \mathrm{a}$ & $<0.001$ & 8.24 & 5.50 & 36.74 \\
\hline V128 & 1223 & 2-Pentylfuran & $3777-69-3$ & $1.32 \mathrm{~b}$ & $1.48 \mathrm{~b}$ & $0.90 \mathrm{a}$ & 1.14ab & 0.008 & $0.71 \mathrm{ab}$ & $0.76 \mathrm{~b}$ & $0.60 \mathrm{a}$ & $0.65 \mathrm{ab}$ & 0.020 & 12.03 & 133.1 & 3.75 \\
\hline V129 & 1294 & (E)-2-Pentenylfuran & 70424-14-5 & $0.37 \mathrm{a}$ & $0.89 \mathrm{~b}$ & $0.38 \mathrm{a}$ & $0.18 \mathrm{a}$ & $<0.001$ & $0.56 \mathrm{a}$ & $0.38 \mathrm{a}$ & $0.47 a$ & $0.35 a$ & 0.087 & 15.10 & 0.28 & 18.63 \\
\hline V130 & 1664 & Estragole & $140-67-0$ & $0.04 a$ & $0.05 a$ & $0.05 a$ & - & 0.536 & 0.02 & - & - & - & - & - & - & - \\
\hline V131 & 1728 & Naphthalene & $91-20-3$ & $0.13 a$ & $0.21 \mathrm{~b}$ & $0.26 \mathrm{~b}$ & $0.24 \mathrm{~b}$ & 0.001 & - & - & - & - & - & - & - & - \\
\hline V132 & 1835 & 2-Methylnaphthalene & $91-57-6$ & $0.01 \mathrm{a}$ & $0.01 \mathrm{a}$ & $0.02 a$ & $0.05 \mathrm{~b}$ & 0.001 & - & - & - & - & - & - & - & - \\
\hline
\end{tabular}

$\mathrm{V}=$ volatiles; LRI = linear retention index; "-" = no peak detected. Different letters within either rind samples or flesh samples indicate significant differences according to ANOVA and Tukey's HSD 
There were 17 volatiles that were not present in all tested cultivars, four of which were exclusive to specific cultivars including estragole $(0.02 \%)$ present only in Captivation, 3-pentanol $(0.01 \%)$ and $\alpha$-terpineol $(<0.01 \%)$ in Exclamation, and $(E, Z)-3,5$-octadien2-one $(0.05 \%)$ in Fascination. Of those volatiles, 3-pentanol and (E,Z)-3,5-octadien-2one have never been reported in watermelon. Other volatiles ( 0.01 to $0.7 \%$ peak area) found in watermelon for the first time and recovered from all four cultivars included $(E, E)-2,6$-nonadienal $(0.06-0.19 \%)$, methanol (0.22-0.24\%), 3-nonen-2-one $(0.11-0.17 \%)$, and methanethiol $(0.24-0.42 \%)$. The remaining unreported volatiles had abundance below $0.1 \%$, such as 2-octanone $(0.04-0.06 \%)$, 2-undecanone (0.06-0.09\%), dihydromyrcenol $(0.03-0.09 \%)$, heptanoic acid $(0.02-0.04 \%)$, and dimethyl disulfide $(0.02-0.03 \%)$, to name a few.

\subsection{Rind and Flesh Volatile Comparison with PCA and Heat Map}

Differences between watermelon flesh and rind were observed in the number of volatile compounds recovered, their total peak area, and the types that were most abundant (Table 1). The differences in volatile abundance between samples is partially due to the samples being from different cultivars or being from different parts of the fruit (flesh or rind). There was a cultivar effect and fruit part effect for 66 and 69 of the volatiles, respectively (Table 1). Of the dominant volatiles in the flesh and rind samples, the abundance of hexanal, (E)-2-nonenal (except in Captivation), 6-methyl-5-hepten-2-one decreased in rind samples compared to flesh samples, while (Z)-3-nonen-1-ol, (E,Z)-2,6-nonadienal (except in Excursion), (Z)-6-nonenal (except in Excursion), nonanal (except in Excursion), $(Z, Z)-3,6-$ nonadien-1-ol, and acetaldehyde increased in rind samples. Two-way ANOVA revealed that the interaction of cultivar and fruit part had a significant effect on the volatile abundance of 59 volatiles (Table 1 ).

PCA was conducted to visualize the relationship between volatiles in watermelon rind and flesh samples. PCA was conducted with all 132 volatiles as loading values and eight samples as score values. Then, all less important variables $\left(\cos ^{2}<0.5\right)$ were removed. Less important variables meant those 26 volatiles common occurred in watermelon samples. A total of 106 volatiles were selected $\left(\cos ^{2} \geq 0.5\right)$ and a new PCA was performed. As shown in Figure 2, the first two components accounted for $81.85 \%$ of the total variance. The first principal component (PC1) explained $62.09 \%$ of the total variance, which was the major component to differentiate samples.

Four watermelon flesh samples were separated on the positive side of PC1. These four flesh samples contained high amounts of 54 volatiles, which included 35 major ones $\left(\cos ^{2} \geq 0.8\right)$. These major volatiles were nine aldehydes (V12, V13, V16, V19, V21, V22, V30, V31, V32), eight alcohols (V35, V37, V47, V48, V52, V54, V61, V66), six ketones (V73, V74, V77, V79, V81, V82), nine terpenes/terpenoids (V89, V96, V98, V102, V104, V105, V106, V107, V108), and two lactones (V117, V118). Three watermelon rind samples (Captivation, Exclamation, and Excursion) were separated on the negative side of PC1, possessing high amounts of 28 volatiles. The eight major ones $\left(\cos ^{2} \geq 0.8\right)$ were V10 (E-3-hexenal), V11 (Z-3-hexenal), V42 (Z-2-penten-1-ol), V55 (nonanol), V64 (3-ethyl-3undecanol), V95 (menthol), V126 (benzothiazole), and V131 (naphthalene). Fascination Rind was markedly separated from other samples on the positive side of PC2, which contained a high amount of 21 volatiles. The major five $\left(\cos ^{2} \geq 0.8\right)$ were V34 (methanol), V80 (2-nonanone), V87 ( $\beta$-myrcene), V110 (ethyl Acetate), and V112 (hexyl acetate). The PCA biplot clearly showed the major difference between watermelon rind and flesh volatile composition. Variety difference within flesh was barely identified in the PCA biplot with the first two PCs. Similarly, no obvious separation of three rind samples (Captivation, Exclamation, and Excursion) was observed. 


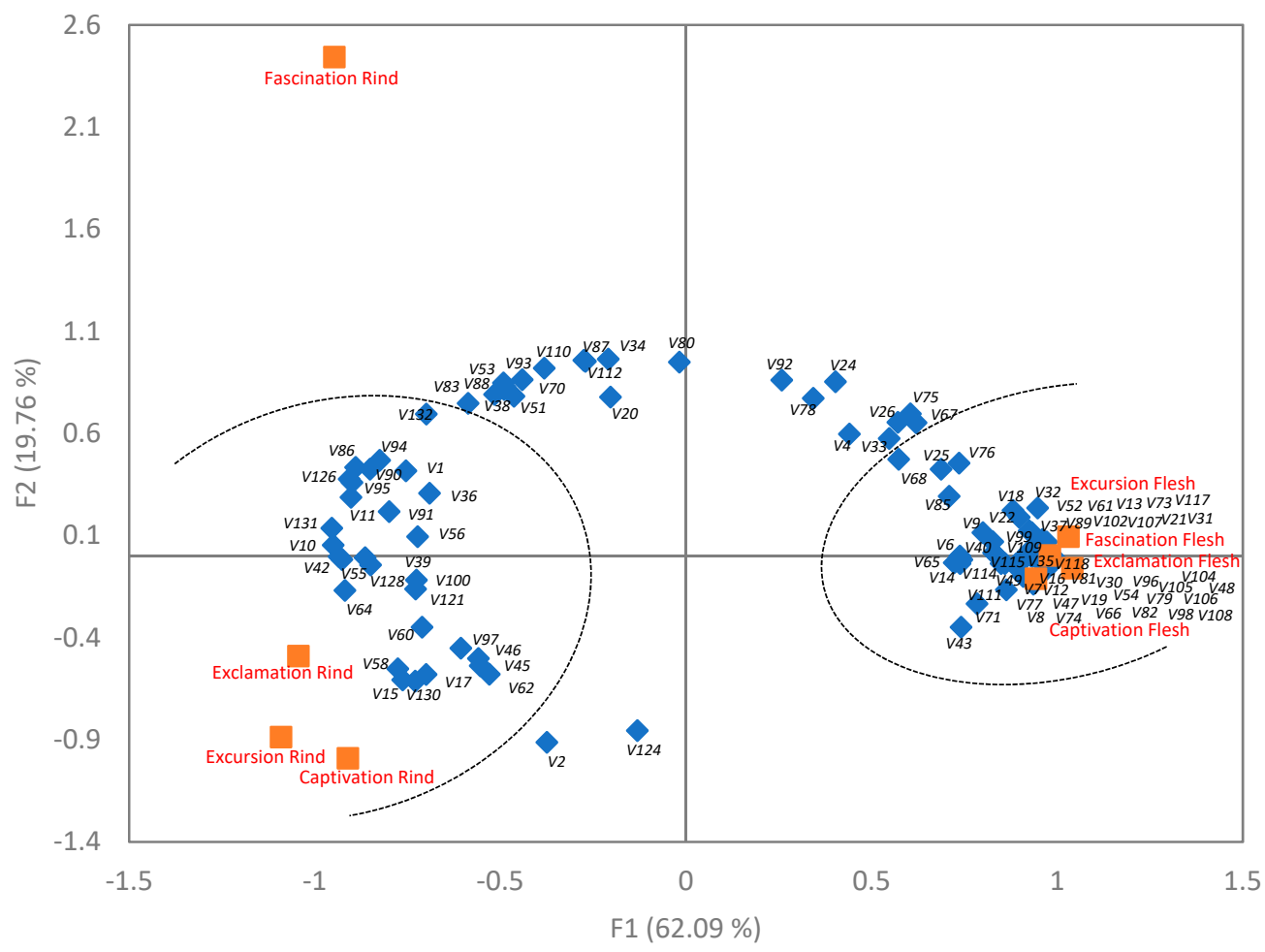

Figure 2. Principal component analysis (PCA) biplot using eight watermelon samples as score values and 106 selected volatiles ( $\%$ peak area, $\cos ^{2}>0.5$ ) as loading values. Rectangle markers indicate watermelon rind and flesh samples, and diamond markers indicate volatiles. According to XLSTAT PCA output of "squared cosines of the variables" and "squared cosines of the observations", two oval circles with dot lines were created on the basis of variables (volatiles) and observations (samples), which were significantly separated from other samples at either the positive or the negative side of PC1. Volatile compound names for V1-V132 could be found in Table 1. V = volatiles.

Heat map cluster analysis was used to visualize the differences and commonalities of the relative percentage abundances of volatiles recovered from the flesh and rind samples (Figure 3). The samples were grouped into two main clusters, one included all four flesh samples and the other included all four rind samples. Within the flesh cluster, Fascination and Exclamation were grouped together and separated from Excursion and Captivation, which were also separated. Within the rind cluster, Excursion and Exclamation were grouped together and separated from Captivation and Fascination, which were also separated. A total of 55 volatiles were selected to differentiate these samples. The main flesh cluster was positively correlated with 31 volatiles, which included an assortment of aldehydes, alcohols, ketones, terpenes/terpenoids, and lactones, while the main rind cluster showed a negative correlation with those volatiles. Captivation flesh showed positive correlations with benzaldehyde, 1-penten-3-one, $(E)$-2-pentenal, $(E, E)$-2,6-nonadienal, and hexanoic acid compared to the flesh of other cultivars, which were negatively correlated to those. A total of 24 volatiles were used to distinguish rind compared to flesh. The rind cluster had more positive correlations with the nine-carbon aldehydes and alcohols that greatly represent watermelon flavor compared to the flesh cluster overall, despite a lesser variation in volatiles in the main rind cluster. Fascination rind was positively correlated with less nine-carbon alcohols compared to Excursion rind. Captivation and Exclamation rinds shared the most negative correlations as well as the most positive correlations with nine-carbon aldehydes. The results were consistent with PCA (Figure 2). 


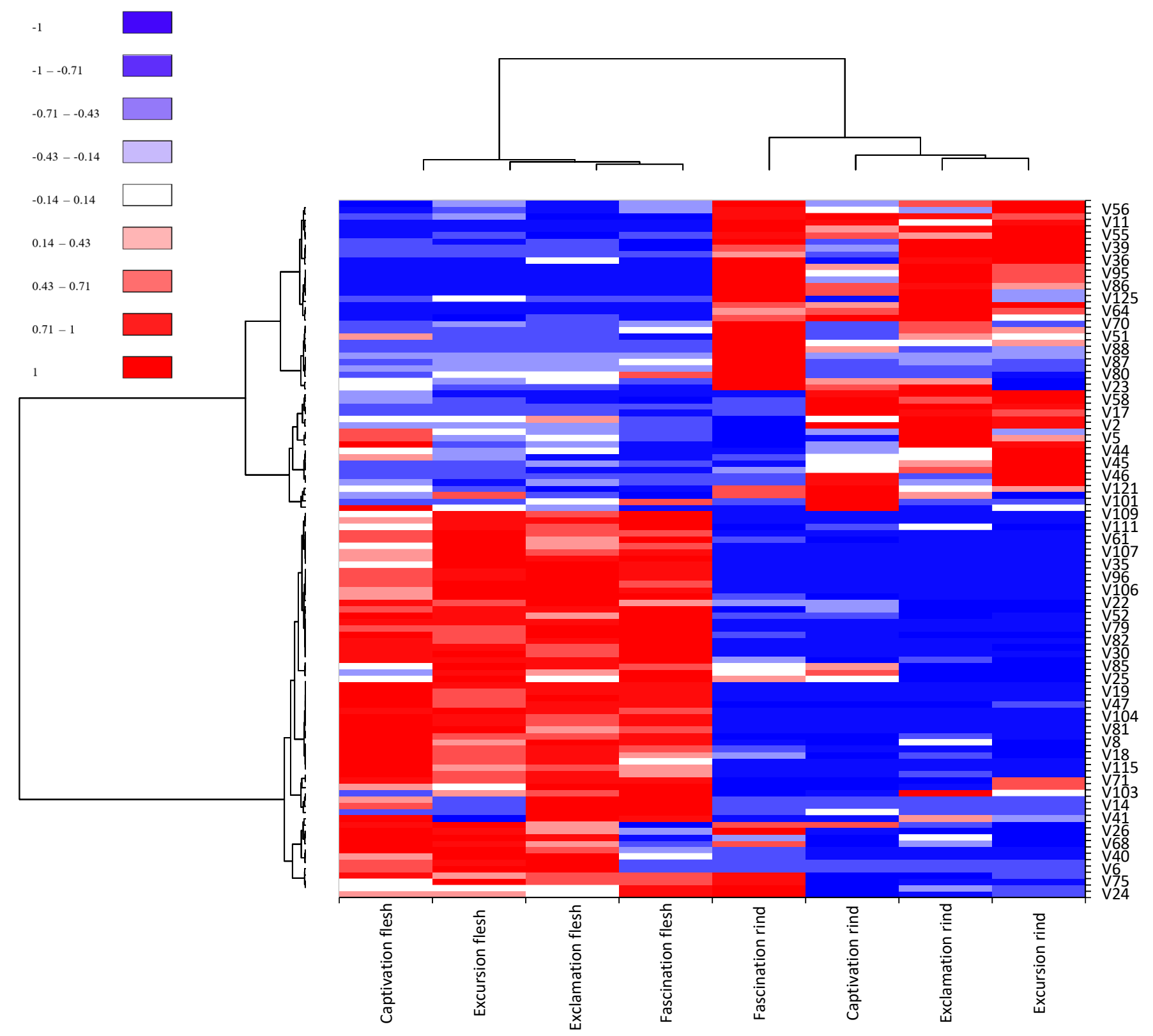

Figure 3. Heat map of 55 selected volatiles ( $\%$ peak area, filtration interquartile range threshold $=0.01$ ) in the rinds and fleshes of Captivation, Exclamation, Excursion, and Fascination watermelons. Volatile compound names for V1-V132 could be found in Table $1 . \mathrm{V}=$ volatiles.

\section{Discussion}

The most abundant grouped volatiles in rind and flesh were aldehydes and alcohols, predominantly volatile nine-carbon compounds. Though volatiles in rind have not yet been reported, the findings of flesh volatiles were consistent with literature in that the most abundant volatile compounds in watermelon flesh are known to be nine-carbon aldehydes and alcohols [29]. Other grouped compounds such as ketones, terpenes, esters, lactones, acid, sulfur compounds, furans, aromatic, and hydrocarbons have also been reported $[1,29]$. Though the identification of these compounds depending on genotypes, growing conditions, and analytical method.

Though diverse groups of compounds occur in watermelon flesh, it has been established that the saturated and unsaturated nine-carbon aldehydes and alcohols are derived from fatty acids, especially polyunsaturated linoleic (C18:2) and linolenic (C18:3). C18:2 
and C18:3 are the main precursors of most fruit aroma volatiles via $\alpha$ - and $\beta$-oxidation in intact fruits, or the LOX pathway in fresh-cut fruits and juices [29]. Nevertheless, studies on the impact of genotypes and metabolism pathway of volatile aroma compounds in watermelon are scarce, though a few studies have reported the volatile compounds derived from the degradation of carotenoid pigments. Consequently, genotypic variation in carotenoid composition underscores differences in the terpenoic and norisoprenoid volatile profiles [1]. Hundreds of volatiles have been identified in watermelon.

Nine-carbon aldehydes and alcohols, such as (Z,Z)-3,6-nonadien-1-ol, (Z)-3-nonenal, $(Z, Z)-3,6$-nonadienal, and (Z)-3-nonen-1-ol, have been identified to be the most important to watermelon aroma as they imparted melon, fresh, sweet, and green notes [32] The most potent volatile compounds determined in watermelon were $(E, Z)-2,6$-nonadienal, (Z)-6-nonenal, (E)-2-nonenal, $(E, Z)$-3,6-nonadien-1-ol, $(Z)$-2-nonenal, $(E, E)$-2,4-nonadienal, nonanal, $(E)-2-n o n e n-1-o l$, and $(E, Z)-2,6$-nonadien-1-ol according to their odor-active values [3]. Another study also indicated nine-carbon aldehydes, alcohols, and their esters were identified as main compounds in watermelon; controversially, ethyl acetate, acetaldehyde, tetradecanoic acid, and methyl acetate were found the most abundant components [33]. In a consumer acceptance study of freshly cut watermelon, high ratings for liking of overall quality were associated with high concentrations of $(E)$-2-nonen-1-ol, (Z)-6-nonen-1-ol, $(E)$ 2-nonenal, and (Z)-6-nonenal. A decrease in some alcohols by $50-80 \%$ and some aldehydes by $\sim 90 \%$ occurred over an eight day storage period, which resulted in decreased odor detection of freshness, green, cucumber, and watermelon aromas [34]. The decline in volatiles and sensory characteristics over a time was also observed by other researchers $[35,36]$.

In addition to nine-carbon aldehydes and alcohols, several other compounds from minor groups might be important to the aroma of watermelon flesh, though their contribution is under-explored. For example, 6-methyl-5-hepten-2-one, the most abundant ketone in watermelon flesh (77.3-83.5\% of the total ketones in this study), can contribute green, musty, fruit odors $[3,28]$. Increased concentration of some volatiles such as acetophenone might contribute to off odors [34]. Terpenes/terpenoids such as $\beta$-ionone can contribute floral notes (violet-like) to watermelon [3], along with other terpenes/terpenoids such as linalool, citral, and geraniol; however, their contribution is not well defined. Esters are well known to contribute fruity notes to many fruits. Esters, however, are not the major grouped compounds in watermelon and only several esters have been identified in this study as well in literature [28,37]. Our current study identified two lactones. $\gamma$-Nonalactone had low peak abundance, but potentially, it could contribute sweet, creamy notes to watermelon. Volatile organic acids are barely found in watermelon literature. One of five acids identified in this study, hexanoic acid, might contribute fatty, cheesy notes to watermelon, though these notes are minimal for watermelon organoleptic characteristics. Three sulfur compounds were identified in this study, while many more have been identified by other researchers $[28,38,39]$. Sulfur compounds are considered off-notes for watermelon, especially processed watermelon juice [28,39].

In the present study, rind and flesh samples of different varieties differed significantly in their total volatile abundance. Results also showed that different watermelon varieties were differentiated by their volatile profile percentages of aldehydes, alcohols, and ketones (especially for flesh), indicating that volatiles are linked to the genetics of watermelon. The findings are in line with other researchers stating significant variation in the amounts and profiles of volatile among genotypes and fruit fractions $[3,40,41]$. Literature specifically pertaining to watermelon volatile variety difference is very limited. The total volatile content of five watermelon varieties was found to vary by a factor of up to one time [3]. Additionally, different parts of the watermelon contain different bioactive compound concentrations and antioxidant activities [41]; however, volatiles in different parts of watermelon have not yet been analyzed. Volatiles in the rind and flesh of four watermelon cultivars (Captivation, Exclamation, Excursion, and Fascination) were identified in this study for the first time, though their sensory profiles have been reported previously [30]. Horticultural traits of Captivation, Exclamation, and Fascination have also been reported [42-44]. 
Watermelon rind has advantages of high citrulline content compared to flesh [14], and contained major volatile compounds of nine-carbon aldehydes and alcohols as identified in this study. The results of the major nine-carbon volatiles in rind from the current study bring in a new potential use of watermelon rind; for example, watermelon rind might be used in highly nutritive plant waters, which could be marketed as consumer hydration options, with fresh, green flavor notes imparted by watermelon rind [45]. It should be pointed out that our previous consumer test shows that high amounts of watermelon rind imparts excessive green notes and off-flavor, negatively correlating with consumer hedonic rating [45]. The previous study also shows refreshing perception of watermelon juice blended with rind [45]. Combined our previous sensory study with this rind volatile study, it could be deduced that watermelon rind has the potential to impart refreshing perception in food products due to its high nine-carbon volatile content. Fruit volatiles are mainly association with aroma contribution, instead of other functions such as refreshing perception. The relationship between watermelon rind volatiles and refreshing perception is an interesting area, which should be further verified in future studies.

\section{Conclusions}

Volatile profiles of four watermelon rinds were investigated for the first time in this study. Both watermelon rind and flesh volatile profiles were predominantly nine-carbon aldehydes and alcohols. Fewer volatile compounds were recovered from watermelon rind compared to flesh, and volatiles were much lower in abundance in the rind. Variety difference for both rind and flesh was observed, with volatiles contents notably ranging two times within four rinds. These results imply that rind has lower volatile intensity, consequently having bland aromas, compared to flesh. The properties of bland aroma are ideal for using as a food ingredient in different food products. On the other hand, the "little" but "dominated" nine-carbon volatiles in the rind associated with fresh, green, and melon-like aroma have potential to make positive contributions to the food products, especially beverages; although, further studies related to aroma-active compounds are desirable to confirm the rind volatile aroma contribution. The finding provides knowledge that watermelon rind could be a promising supplemental ingredient for food and beverages for either using its bland aroma or its volatiles, potentially contributing to flavor, which depends on how it is used and types of its final products.

Author Contributions: J.R. carried out the study, performed data analysis and interpretation, and wrote the manuscript. X.D. designed and supervised the study, aided with data analysis and interpretation, and wrote and finalized the manuscript. All authors have read and agreed to the published version of the manuscript.

Funding: This research project was funded by the National Watermelon Promotion Board and the grant ID\# was 00704263.

Institutional Review Board Statement: Not applicable.

Informed Consent Statement: Not applicable.

Data Availability Statement: Our data are available for review.

Acknowledgments: The authors thank Mindy Davila (Department of Nutrition and Food Sciences, Texas Woman's University, Denton, TX, USA) for analyzing data with PCA and heat map using XLSTAT software. The authors thank Russell Wallace (Horticultural sciences, Texas A\&M AgriLife Research \& Extension Center, Lubbock, TX, USA) provided watermelon samples.

Conflicts of Interest: The authors declare no conflict of interest.

\section{References}

1. Kyriacou, M.C.; Leskovar, D.I.; Colla, G.; Rouphael, Y. Watermelon and melon fruit quality: The genotypic and agro-environmental factors implicated. Sci. Hortic. 2018, 234, 393-408. [CrossRef]

2. Rico, X.; Gullón, B.; Alonso, J.L.; Yáñez, R. Recovery of high value-added compounds from pineapple, melon, watermelon and pumpkin processing by-products: An overview. Food Res. Int. 2020, 132, e109086. [CrossRef] [PubMed] 
3. Liu, Y.; He, C.; Song, H. Comparison of fresh watermelon juice aroma characteristics of five varieties based on gas chromatographyolfactometry-mass spectrometry. Food Res. Int. 2018, 107, 119-129. [CrossRef] [PubMed]

4. Kassim, M.A.; Hussin, A.H.; Meng, T.K.; Kamaludin, R.; Zaki, M.S.I.M.; Zakaria, W.Z.E.W. Valorisation of watermelon (Citrullus lanatus) rind waste into bioethanol: An optimization and kinetic studies. Int. J. Environ. Sci. Technol. 2021, e033105. [CrossRef]

5. Romdhane, M.B.; Haddar, A.; Ghazala, I.; Jeddou, K.B.; Helbert, C.B.; Ellouz-Chaabouni, S. Optimization of polysaccharides extraction from watermelon rinds: Structure, functional and biological activities. Food Chem. 2017, 216, 355-364. [CrossRef] [PubMed]

6. Rambabu, K.; Bharath, G.; Hai, A.; Luo, S.; Liao, K.; Abu Haija, M.; Banat, F.; Naushad, M. Development of watermelon rind derived activated carbon/manganese ferrite nanocomposite for cleaner desalination by capacitive deionization. J. Clean. Prod. 2020, 272, 122626. [CrossRef]

7. Liu, C.; Ngo, H.H.; Guo, W. Watermelon rind: Agro-waste or superior biosorbent? Appl. Biochem. Biotechnol. 2012, 167, 1699-1715. [CrossRef] [PubMed]

8. Yang, Y.; Xu, P.; Dong, S.; Yu, Y.; Chen, H.; Xiao, J. Using watermelon rind and nitrite-containing wastewater for electricity production in a membraneless biocathode microbial fuel cell. J. Clean. Prod. 2021, 307, e127306. [CrossRef]

9. Lakshmipathy, R.; Sarada, N.C. Biosorptive removal of methylene blue from aqueous solution by chemically activated watermelon rind as adsorbent. J. Indian Chem. Soc. 2015, 92, 999-1002.

10. Ho, L.; Ramli, N.F.; Tan, T.; Muhamad, N.; Haron, M.N. Effect of extraction solvents and drying conditions on total phenolic content and antioxidant properties of watermelon rind powder. Sains Malays. 2018, 47, 99-107. [CrossRef]

11. Egbuonu, A.C.C. Comparative assessment of some mineral, amino acid and vitamin compositions of watermelon (Citrullus lanatus) rind and seed. Asian J. Biochem. 2015, 10, 230-236. [CrossRef]

12. Al-Sayed, H.M.A.; Ahmed, A.R. Utilization of watermelon rinds and sharlyn melon peels as a natural source of dietary fiber and antioxidants in cake. Ann. Agric. Sci. 2013, 58, 83-95. [CrossRef]

13. Tarazona-Díaz, M.P.; Viegas, J.; Moldao-Martins, M.; Aguayo, E. Bioactive compounds from flesh and by-product of fresh-cut watermelon cultivars. J. Sci. Food Agric. 2011, 91, 805-812. [CrossRef] [PubMed]

14. Rimando, A.M.; Perkins-Veazie, P.M. Determination of citrulline in watermelon rind. J. Chromatogr. A 2005, 1078, 196-200. [CrossRef]

15. Méndez, D.A.; Fabra, M.J.; Gómez-Mascaraque, L.; López-Rubio, A.; Martinez-Abad, A. Modelling the extraction of pectin towards the valorisation of watermelon rind waste. Foods 2021, 10, 738. [CrossRef]

16. Petkowicz, C.L.O.; Vriesmann, L.C.; Williams, P.A. Pectins from food waste: Extraction, characterization and properties of watermelon rind pectin. Food Hydrocoll. 2017, 65, 57-67. [CrossRef]

17. Simonne, A.; Carter, M.; Fellers, R.; Weese, J.; Wei, C.I.; Smonne, E.; Miller, M. Chemical, physical and sensory characterization of watermelon rind pickles. J. Food Process. Preserv. 2003, 26, 415-431. [CrossRef]

18. Souad, A.M.; Jamal, P.; Olorunnisola, K.S. Effective jam preparations from watermelon waste. Int. Food Res. J. 2012, 19, 1545-1549.

19. Hoque, M.M.; Iqbal, A. Drying of watermelon rind and development of cakes from rind powder. Int. J. Nov. Res. Life Sci. 2015, 2, 14-21.

20. Naknaen, P.; Itthisoponkul, T.; Sondee, A.; Angsombat, N. Utilization of watermelon rind waste as a potential source of dietary fiber to improve health promoting properties and reduce glycemic index for cookie making. Food Sci. Biotechnol. 2016, 25, 415-424. [CrossRef]

21. Olaitan, N.I.; Eke, M.O.; Agudu, S.S. Effect of watermelon (Citrullus lanatus) rind flour supplementation on the quality of wheat based cookies. Int. J. Eng. Sci. 2017, 6, 59-66. [CrossRef]

22. Chakrabarty, N.; Mourin, M.M.; Islam, N.; Haque, A.R.; Akter, S.; Siddique, A.A.; Sarker, M. Assessment of the Potential of Watermelon Rind Powder for the Value Addition of Noodles. J. Biosyst. Eng. 2020, 45, 223-231. [CrossRef]

23. Ho, L.; Che Dahri, N. Effect of watermelon rind powder on physicochemical, textural, and sensory properties of wet yellow noodles. CyTA-J. Food 2016, 14, 465-472. [CrossRef]

24. Badr, S.A.; El-Waseif, M.A.; Ghaly, M.S. Effect of Addition Watermelon Rind Powder on Quality Criteria and Microbial Aspects of Beef Burger Patties during Frozen Storage Periods. J. Food Dairy Sci. 2018, 9, 177-187. [CrossRef]

25. Kumar, P.; Mehta, N.; Malav, O.P.; Kumar Chatli, M.; Rathour, M.; Kumar Verma, A. Antioxidant and antimicrobial efficacy of watermelon rind extract (WMRE) in aerobically packaged pork patties stored under refrigeration temperature $\left(4 \pm 1{ }^{\circ} \mathrm{C}\right) . \mathrm{J}$. Food Process. Preserv. 2018, 42, e13757. [CrossRef]

26. Hasanin, M.S.; Hashem, A.H. Eco-friendly, economic fungal universal medium from watermelon peel waste. J. Microbiol Methods. 2020, 168, e105802. [CrossRef] [PubMed]

27. Erukainure, O.L.; Oke, O.V.; Daramola, A.O.; Adenekan, S.O.; Umanhonlen, E.E. Improvement of the biochemical properties of watermelon rinds subjected to Saccharomyces cerevisae solid media fermentation. Pak. J. Nutr. 2010, 9, 806-809. [CrossRef]

28. Yang, F.; Liu, Y.; Wang, B.; Song, H.; Zou, T. Screening of the volatile compounds in fresh and thermally treated watermelon juice via headspace-gas chromatography-ion mobility spectrometry and comprehensive two-dimensional gas chromatographyolfactory-mass spectrometry analysis. LWT 2020, 137, 11047. [CrossRef]

29. Ilahy, R.; Tlili, I.; Siddiqui, M.W.; Hdider, C.; Lenucci, M.S. Inside and beyond color: Comparative overview of functional quality of tomato and watermelon fruits. Front. Plant. Sci. 2019, 10, e00769. [CrossRef] 
30. Ramirez, J.L.; Du, X.; Wallace, R.W. Investigating sensory properties of seven watermelon varieties and factors impacting refreshing perception using quantitative descriptive analysis. Food Res. Int. 2020, 138, e109681. [CrossRef]

31. Scott, G.; Williams, C.; Wallace, R.W.; Du, X. Exploring plant performance, fruit physicochemical characteristics, volatile profiles, and sensory properties of day-neutral and short-day strawberry cultivars grown in Texas. J. Agric. Food Chem. 2021, 69, 13299-13314. [CrossRef] [PubMed]

32. Yajima, I.; Sakakibara, H.; Ide, J.; Yanai, T.; Hayashi, K. Volatile flavor components of watermelon (Citrullus vulgaris). Agric. Biol. Chem. 1985, 49, 3145-3150. [CrossRef]

33. Pino, J.A.; Marbot, R.; Aguero, J. Volatile components of watermelon (Citrullus ianatus [thunb.] Matsum. et Nakai) fruit. J. Essent. Oil Res. 2003, 15, 379-380. [CrossRef]

34. Mendoza-Enano, M.L.; Stanley, R.; Frank, D. Linking consumer sensory acceptability to volatile composition for improved shelf-life: A case study of fresh-cut watermelon (Citrullus lanatus). Postharvest Biol. Technol. 2019, 154, 137-147. [CrossRef]

35. Saftner, R.; Luo, Y.; McEvoy, J.; Abbott, J.A.; Vinyard, B. Quality characteristics of fresh-cut watermelon slices from non-treated and 1-methylcyclopropene- and/or ethylene-treated whole fruit. Postharvest Biol. Technol. 2007, 44, 71-79. [CrossRef]

36. Xisto, A.L.R.P.; de Barros Vilas Boas, E.V.; Nunes, E.E.; Vilas Boas, B.M.; Guerreiro, M.C. Volatile profile and physical, chemical, and biochemical changes in fresh cut watermelon during storage. Food Sci. Technol. 2012, 32, 173-178. [CrossRef]

37. Fredes, A.; Sales, C.; Barreda, M.; Valcárcel, M.; Roselló, S.; Beltrán, J. Quantification of prominent volatile compounds responsible for muskmelon and watermelon aroma by purge and trap extraction followed by gas chromatography-mass spectrometry determination. Food Chem. 2016, 190, 689-700. [CrossRef]

38. Liu, Y.; He, C.; Song, H.L. Comparison of SPME versus SAFE processes for the analysis of flavor compounds in watermelon juice. Food Anal. Methods 2018, 11, 1677-1689. [CrossRef]

39. Yang, X.; Yang, F.; Liu, Y.; Li, J.; Song, H. Identification of key off-flavor compounds in thermally treated watermelon juice via gas chromatography-olfactometry-mass spectrometry, aroma recombination, and omission experiments. Foods $2020,9,227$. [CrossRef]

40. Beaulieu, J.C.; Lea, J.M. Characterization and semiquantitative analysis of volatiles in seedless watermelon varieties using solid-phase microextraction. J. Agric. Food Chem. 2006, 54, 7789-7793. [CrossRef]

41. Tlili, I.; Hdider, C.; Lenucci, M.S.; Riadh, I.; Jebari, H.; Dalessandro, G. Bioactive compounds and antioxidant activities of different watermelon (Citrullus lanatus (thunb.) mansfeld) cultivars as affected by fruit sampling area. J. Food Compos. Anal. 2011, 24, 307-314. [CrossRef]

42. Huber, B.; Hernández, R. Optimizing production of 'Fascination' and 'Carnivor' transplants for grafting using lower daily light integral and higher $\mathrm{CO}_{2}$. Int. Soc. Hortic. Sci. 2021, 1302, e130214. [CrossRef]

43. Trandel, M.A.; Perkins-Veazie, P.; Schultheis, J. Predicting hollow heart incidence in triploid watermelon (Citrullus lanatus). HortScience 2020, 55, 1926-1930. [CrossRef]

44. Bertucci, M.B.; Jennings, K.; Monks, D.W.; Schultheis, J.R.; Perkins-Veazie, P.; Louws, F.; Jordan, D.L. Early Season Growth, Yield, and Fruit Quality of Standard and Mini Watermelon Grafted onto Several Commercially Available Cucurbit Rootstocks. HortTechnology 2018, 28, 459-469. [CrossRef]

45. Ramirez, J.L.; Juma, S.; Du, X. Consumer acceptance of watermelon flesh-rind blends and the effect of rind on refreshing perception. J. Food Sci. 2021, 86, 1384-1392. [CrossRef] [PubMed] 